\title{
Effect of Lipoic Acid on the Biochemical Mechanisms of Resistance to Bortezomib in SH-SY5Y Neuroblastoma Cells
}

\author{
Daniele Tibullo $^{1,2}$ - Cesarina Giallongo ${ }^{1,2}$. Fabrizio Puglisi ${ }^{2} \cdot$ Daniele Tomassoni $^{3}$. \\ Giuseppina Camiolo $^{1}$ - Martina Cristaldi ${ }^{1}$ - Maria Violetta Brundo ${ }^{1}$. \\ Carmelina Daniela Anfuso ${ }^{1}$ • Gabriella Lupo ${ }^{1}$ - Tomaso Stampone ${ }^{4}$ - Giovanni Li Volti ${ }^{1}$. \\ Francesco Amenta $^{5}$ - Roberto Avola ${ }^{1}$ • Vincenzo Bramanti ${ }^{1,4}$
}

Received: 13 March 2017 / Accepted: 25 April 2017

(C) Springer Science+Business Media New York 2017

\begin{abstract}
Neuroblastoma (NB) is an extracranial solid cancer and the most common cancer in infancy. Despite the standard treatment for NB is based on the combination of chemotherapeutic drugs such as doxorubicin, vincristine, cyclophosphamide, and cisplatin, chemoresistance occurs over the time. The aim of the present research was to evaluate the effect of bortezomib (BTZ) $(50 \mathrm{nM})$ on NB cell viability and how lipoic acid (ALA) $(100 \mu \mathrm{M})$ modifies pharmacological response to this chemotherapeutic agent. Cell viability was assessed by ATP luminescence assay whereas expression of oxidative stress marker (i.e., heme oxygenase-1) and endoplasmic reticulum stress proteins was performed by real-time PCR, western blot, and immunofluorescence. Our data showed that BTZ treatment significantly reduced cell viability when compared to untreated cultures (about 40\%). Interestingly, ALA significantly reduced the efficacy of BTZ (about 30\%). Furthermore, BTZ significantly induced heme oxygenase- 1 as a result of increased oxidative stress and such
\end{abstract}

Roberto Avola and Vincenzo Bramanti contributed equally

Roberto Avola

ravola@unict.it

1 Department of Biomedical and Biotechnological Sciences, University of Catania, Via S. Sofia 87, 95100 Catania, Italy

2 Division of Haematology, A.O.U. "Policlinico-Vittorio Emanuele", University of Catania, Via Citelli 6, 95100 Catania, Italy

3 School of Bioscience and Veterinary Medicine, University of Camerino, via Gentile III da Varano, Camerino 62032, Italy

4 Division of Microbiology and Virology, Villa Sofia Hospital, A.O.O.R. "Villa Sofia-Cervello", Piazza Salerno 1, 90146 Palermo, Italy

5 School of Medicinal Sciences and Health Products, University of Camerino, via Madonna delle Carceri 9, Camerino 62032, Italy overexpression was prevented by concomitant treatment with ALA. Similarly, ALA significantly reduced BTZ-mediated endoplasmic reticulum stress as measured by reduction in BiP1 and IRE1 $\alpha$, ERO1 $\alpha$, and PDI expression. In conclusion, our data suggest that BTZ efficacy is dependent on cellular redox status and such mechanisms may be responsible of chemoresistance to this chemotherapeutic agent.

Keywords Neuroblastoma cell cultures $\cdot$ Lipoic acid · Bortezomib $\cdot$ HO- $1 \cdot$ ER-stress $\cdot$ Cancer

\section{Introduction}

Neuroblastoma (NB) is an extracranial solid cancer and the most common cancer in infancy [1]. It is a neuroendocrine tumor, and deriving from the neural crest component of the sympathetic nervous system (SNS), it is capable to switch from the highly aggressive chemoresistant disease to the spontaneous regression [2]. Despite the standard treatment for NB is based on the combination of chemotherapeutic drugs such as doxorubicin, vincristine, cyclophosphamide, and cisplatin, chemoresistance occurs over the time. Therefore, it is important to study and evaluate new therapeutic approaches for the treatment of this cancer.

Bortezomib (BTZ), a reversible inhibitor of the $26 \mathrm{~S}$ proteasome, is used as counteracting drug against the multiple myeloma (MM) [3]. However, recent experimental evidences demonstrated that BTZ treatment is capable to overcome cancer cell resistance in different solid tumors, including NB [4]. In addition, it has been reported a synergistic effect when this drug was used in combination with doxorubicin in vitro [5]. However, BTZ treatment in NB cell lines induces overexpression of heme oxygenase 1 (HO-1), which in turn leads to BTZ resistance [6]. Similarly, our group showed that BTZ-induced 
HO- 1 is involved in resistance to proteosome inhibitor and is involved in genomic instability of MM [7]. Heme oxygenases catalyze the degradation of heme into biliverdin, carbon monoxide (CO), and ferric iron. Such upregulation represents an intrinsic defense mechanism to maintain cellular homeostasis and enhance cell survival [8]. In some cancer cells, HO-1 is considered to play a major role as an essential survival factor, protecting against chemotherapy-induced reactive oxygen species (ROS) increase [9-14]. Interestingly, the role of HO1 in cancer cells has been shown to be cell-specific since in some tumors its upregulation has been shown to be associated with cell cycle arrest and/or death whereas in other malignancies it was associated with tumor progression and survival [15, 16]. Therefore, it is important to evaluate the effects of pharmacological interventions causing a significant modification of redox status and resulting in a modification of cellular response to chemotherapy. Furthermore, it is well known that (+)lipoic acid (ALA) is a neuroprotective and antioxidant agent able to scavenge ROS, to regenerate endogenous antioxidants as well as to promote glutathione synthesis and it also serves as metals chelator $[17,18]$. It is well known that oxidative stress is implicated in the development and evolution of a lot of diseases and there are many factors related to oxidative stress as well as to the development of several pathologies. In this regard, previous reports demonstrated that ALA prevented cell death induced by drugs depleting glutathione in NB cells [17]. In fact, it is well known that oxidative stress is implicated in the development and evolution of a lot of diseases and there are many factors related to oxidative stress as well as to the development of several pathologies. In addition, ROS are reactive molecules derived from the natural byproduct of the oxygen metabolism and having significant roles in the homeostasis and in the cell signaling. Moreover, among the antioxidants of the latest generation, lipoic acid is capable to fulfill many important functions of "scavenger" of hydroxyl radicals, hypochlorous acid, of oxygen singlet, and of peroxyl radicals.

Finally, the aim of the present study was to study and evaluate the effect of ALA on NB redox balance and how such effect may impact on NB response to BTZ.

\section{Material and Methods}

\section{Cell Cultures and Treatments}

NB cell lines were cultured in DMEM supplemented with $10 \% \mathrm{FBS}$ and $1 \%$ penicillin/streptomycin at $37{ }^{\circ} \mathrm{C}$ and $5 \%$ $\mathrm{CO}_{2}$. ALA was added $24 \mathrm{~h}$ before the addition of BTZ $(50 \mathrm{nM})$ for ALA/BTZ combined treatment. For estimation of the effect of BTZ on ER-stress markers and HO-1 expression, NB cells were seeded in six-well culture plate at density $5 \times 10^{5}$ cell per well and treated with BTZ alone and in combination with $5 \mathrm{mM}$ 4-sodium phenylbutyrate (4PBA, Sigma-Aldrich, Milan, Italy) for 6 and $24 \mathrm{~h}$, with $10 \mu \mathrm{M}$ thapsigargin (Santa Cruz Biotechnology) alone and in combination with $5 \mathrm{mM} 4$-PBA for $24 \mathrm{~h}$. For viability assay, NB cells were seeded on 96-well culture plate (Eppendorf, Milan, Italy) at density $1 \times 10^{4}$ cell per well, and subsequently treated with $100 \mu \mathrm{M}$ of ALA. After $24 \mathrm{~h}, 50 \mathrm{nM}$ BTZ alone and in combination with ALA was added to cell cultures for $24 \mathrm{~h}$. All agents were diluted directly in cell culture medium.

\section{Cell Viability Assay}

Cell viability was assessed using ATPlite 1step assay (PerkinElmer, Milan, Italy) according to the manufacturers' protocol. Briefly, the 96-well black culture plate was taken from the incubator and equilibrated at room temperature for $30 \mathrm{~min}$. Subsequently, to each well containing $100 \mu \mathrm{l}$ of the cell suspension $\left(5 \times 10^{5}\right.$ cells $\left./ \mathrm{ml}\right), 100 \mu \mathrm{l}$ of reconstituted reagent was added and the plate was shaken for $20 \mathrm{~min}$ at $700 \mathrm{rpm}$ using orbital shaker (Stuart Scientific, Staffordshire, UK). The luminescence was measured using Victor3 (PerkinElmer, Milan, Italy). Viability of the cells was expressed as percentage of vitality of untreated cells.

\section{Gene Expression Analysis by Real-Time PCR (qRT-PCR)}

RNA was extracted by Trizol reagent (Invitrogen, Carlsbad, CA, USA). First strand cDNA was then synthesized with Applied Biosystem (Foster City, CA, USA) reverse transcription reagent $[16,19]$. HO-1 mRNA expression was assessed by TaqMan Gene Expression, Applied Biosystem and quantified using a fluorescence-based real-time detection method by 7900HT Fast Real Time PCR System (Life technologies, Carlsbad, CA, USA). For each sample, the relative expression level of HO-1 (Hs01110250_m1), Ire 1 alpha (Hs00980095_m1), and BIP/ GRP78 (Hs00607129_gH) mRNA was normalized using GAPDH (Hs02758991_g1) as an invariant control [20].

\section{Western Blot Analysis}

Briefly, for western blot analysis, $30 \mu \mathrm{g}$ of protein was loaded onto a $12 \%$ polyacrylamide gel MiniPROTEAN® TGX ${ }^{\mathrm{TM}}$ (BIO-RAD, Milan, Italy) followed by electrotransfer to nitrocellulose membrane TransBlot ${ }^{\circledR}$ Turbo $^{\mathrm{TM}}$ (BIO-RAD, Milan, Italy) using TransBlot ${ }^{\circ}$ SE Semi-Dry Transfer Cell (BIORAD, Milan, Italy). Subsequently, membrane was blocked in Odyssey Blocking Buffer (Licor, Milan, Italy) for $1 \mathrm{~h}$ at room temperature. After blocking, membrane was three times washed in phosphate-buffered saline (PBS) for $5 \mathrm{~min}$ and incubated with primary antibodies against HO-1 (1:1000) (anti-rabbit, Cat. No. BML-HC3001-0025, Enzo Life Sciences, Milan, Italy), BiP (1:1000) (anti-rabbit, Cat. No. 3177S, Cell 
Signaling Technology, Milan, Italy), Iron Responsive Element $1 \alpha(\operatorname{IRE} 1 \alpha)(1: 1000)$ (anti-rabbit, Cat. No. 3294S, Cell Signaling Technology, Milan, Italy), PDI (1:1000) (antirabbit, Cat. No. 5683S, Cell Signaling Technology, Milan, Italy), ERO (1:1000), and $\beta$-actin (1:1000) (anti-mouse, Cat. No. 4967S, Cell Signaling Technology, Milan, Italy), overnight at $4{ }^{\circ} \mathrm{C}$ [21]. Next day, membranes were three times washed in PBS for $5 \mathrm{~min}$ and incubated with infrared antimouse IRDye800CW (1:5000) and anti-rabbit IRDye700CW secondary antibodies (1:5000) in PBS/0.5\% Tween-20 for $1 \mathrm{~h}$ at room temperature $[22,23]$. All antibodies were diluted in Odyssey Blocking Buffer. The blots were visualized using Odyssey Infrared Imaging Scanner (Licor, Milan, Italy) and protein levels were quantified by densitometric analysis. Data were normalized to $\beta$-actin expression $[24,25]$.

\section{Immunofluorescence}

Cells were grown directly on coverslips before immunofluorescence. After washing with PBS, cells were fixed in $4 \%$ paraformaldehyde (Sigma-Aldrich, Milan, Italy) for $20 \mathrm{~min}$ at room temperature [26, 27]. Subsequently, cells were incubated with primary antibody against HO-1 (anti-rabbit, Cat. No. BMLHC3001-0025, Enzo Life Sciences, Milan, Italy) at dilution 1:200 and against $\beta$-actin (anti-mouse, Cat. No. 4967S, Cell Signaling Technology, Milan, Italy) at dilution 1:200, overnight at $4{ }^{\circ} \mathrm{C}$. Next day, cells were washed three times in PBS for 5 min and incubated with secondary antibodies: TRITC (anti-mouse, Cat. No. sc-3796, Santa Cruz Biotechnology) at dilution 1:200, and FITC (anti-rabbit, Cat. No. sc-2012, Santa Cruz Biotechnology, Santa Cruz, CA, USA) at dilution 1:200 for $1 \mathrm{~h}$ at room temperature. The slides were mounted with medium containing DAPI (4', 6diamidino-2phenylindole, Santa Cruz Biotechnology, Santa Cruz, CA, USA) to visualize nuclei [28]. The fluorescent images were obtained using a Zeiss Axio Imager Z1 Microscope with Apotome 2 system (Zeiss, Milan, Italy). As a control, the specificity of immunostaining was verified by omitting incubation with the primary or secondary antibody. Immunoreactivity was evaluated taking into account the signal-to-noise ratio of immunofluorescence.

\section{Statistical Analysis}

Statistical analyses were made by Prism Software (Graphpad Software Inc., La Jolla, CA, USA), (Graphpad Prism, data analysis software, RRID: rid 000081). The data are expressed as mean \pm SEM. Statistical analysis was carried out by ANOVA test: it is used to compare the means of more than two samples. The significance of differences between means was analyzed by analysis of variance. A $p$ value of less than $0.05(* p<0.05)$ was accepted as statistically significant between experimental and control groups.

\section{Results}

\section{ALA Effects in BTZ-Induced Cytotoxicity in Neuroblastoma Cell Lines}

We observed that BTZ $50 \mathrm{nM}$ exhibited cytotoxicity in SHSY5Y ( $p<0.0001)$ (Fig. 1). Moreover, we tested the effects of ALA on SH-SY5Y cell proliferation alone or in combination with BTZ. Our data also showed that ALA alone had no significant effect on cell viability, whereas co-treatment with BTZ significantly decreased the cytotoxic effect of BTZ $(p<0.0001)$ (Fig. 1).

\section{ALA Reduces HO-1 Expression Induced by BTZ and Increase Its Nuclear Translocation}

In order to evaluate cellular oxidative stress response after BTZ treatment alone or in combination with ALA, we evaluated HO-1 expression. We observed a significant increase of HO-1 mRNA following treatment with BTZ $50 \mathrm{nM}(p<0.0001)$ (Fig. 2a). The evaluation of HO-1 protein expression by western blot assay further confirmed such effect (Fig. 2b, c). Interestingly, these set of experiments suggested a possible cleavage of HO-1 protein (Fig. 2b), which it is known to allow HO-1 translocation into the nuclear compartment where it may exerts its non-canonical functions (i.e., genetic instability, transcriptional activation of antioxidant genes). In order to confirm such evidence, we studied the nuclear localization of HO-1 in neuroblastoma cells by structured Illumination ZEISS Apotome 2 microscopy. We observed an increase of protein expression and nuclear localization signal of HO-1 in neuroblastoma cells after treatment with BTZ

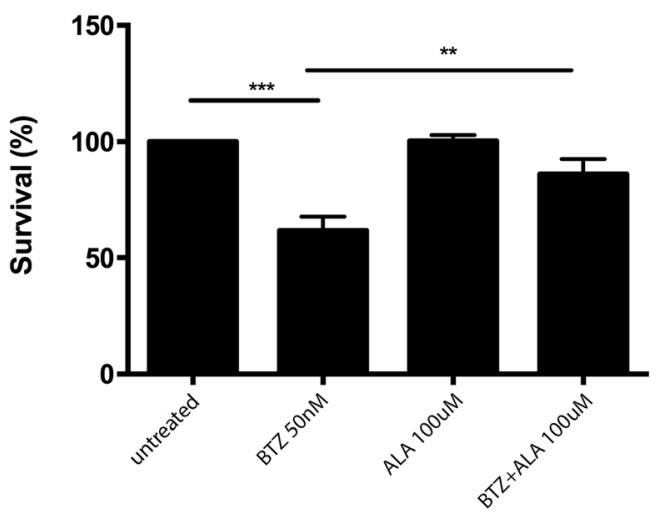

Fig. 1 Determination of cell viability of NB cell lines. ATPLite analysis for cell viability evaluation of $\mathrm{NB}$ cell lines untreated, treated with BTZ $(50 \mathrm{nM})$ alone, treated with ALA $(100 \mu \mathrm{M})$ alone and treated with BTZ + ALA in combination. The data are expressed as mean \pm SEM (standard error of the mean). The significance of differences between means was analyzed by analysis of variance. $(* p<0.05)(* * p<0.01)(* * * p<0.001)$ 
Fig. 2 Evaluation of HO-1 gene and protein expression on NB cell lines. a Evaluation of HO-1 gene expression after treatment with ALA $(100 \mu \mathrm{M})$ alone, with BTZ $(50 \mathrm{nM})$ alone, and with BTZ + ALA in combination, in NB cell lines. b Evaluation of HO-1 protein expression after treatment with ALA $(100 \mu \mathrm{M})$ alone, with $\mathrm{BTZ}(50 \mathrm{nM})$ alone, and with $\mathrm{BTZ}+\mathrm{ALA}$ in combination, in NB cell lines. The data are expressed as mean $\pm \mathrm{SEM}$. The significance of differences between means was analyzed by analysis of variance. $(* p<0.05)$ $(* * p<0.01)(* * * p<0.001)$ a

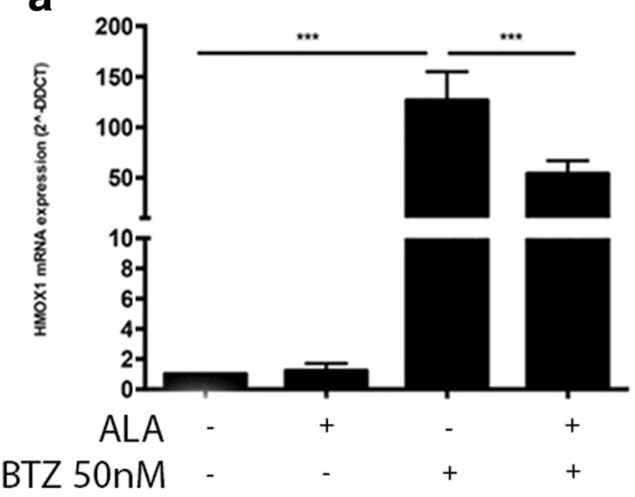

b

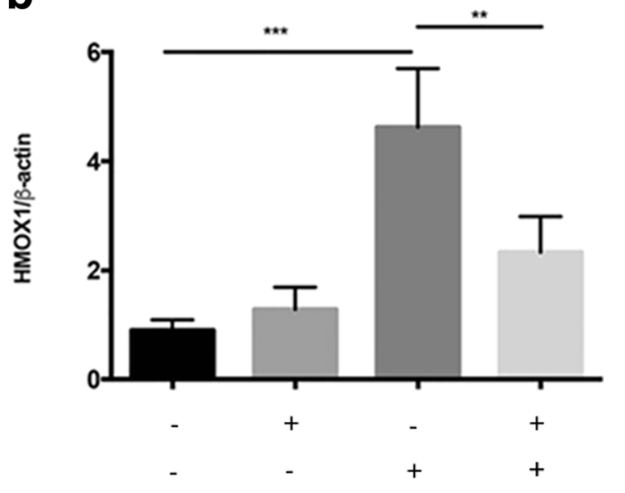

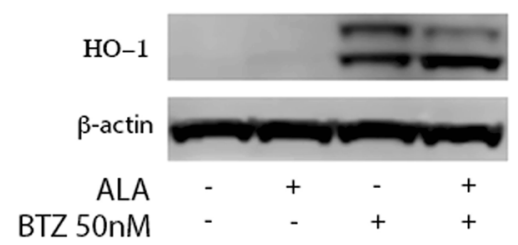

(Fig. 3) compared with untreated cells. Cytoplasmic localization of HO-1 was disrupted when BTZ and ALA were used in combination (Fig. 3). Moreover, ALA did not induce HO-1 expression, but in combination with BTZ, ALA was able to reverse BTZ-induced HO-1 expression and promoted its nuclear localization (Fig. 3). In order to confirm ER-stress-induced HO-1 expression, we performed the real-time PCR of the UPR related genes after treatment with thapsigargin alone or in combination with a chemical chaperon 4-PBA. We observed that the thapsigargin treatment of neuroblastoma cells induced
UPR related genes expressions such as HO-1 and this effects was reverted by 4-PBA (Fig. 4).

\section{ALA Modulates ER-Stress Signaling}

Our data showed that BTZ was able to induce protein expression of ER-stress markers BiP1, IRE1 $\alpha$, ERO $1 \alpha$, and PDI $(p<0.0001)$ compared to control. By contrast, when NB cell lines were exposed to BTZ in combination with ALA, we observed a concomitant reduction of ER-stress protein levels $(p<0.001)$ in respect to BTZ alone (Fig. 5).
Fig. 3 Microscopy analysis of HO-1 localization in NB cell lines. The detection of HO-1 was performed by incubation with anti-rabbit secondary antibody followed by monoclonal antibody conjugated with TRITC (red).

The counter-staining of the cells was performed using the nuclear dye DAPI (blue)
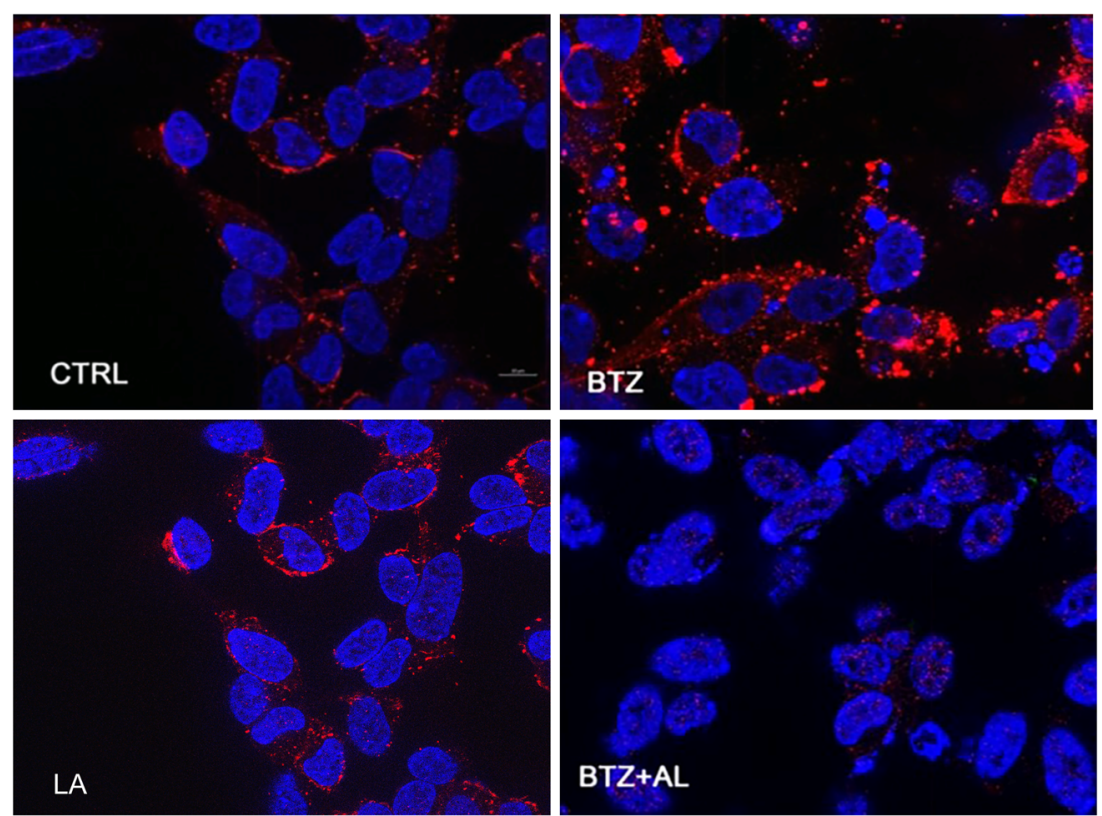

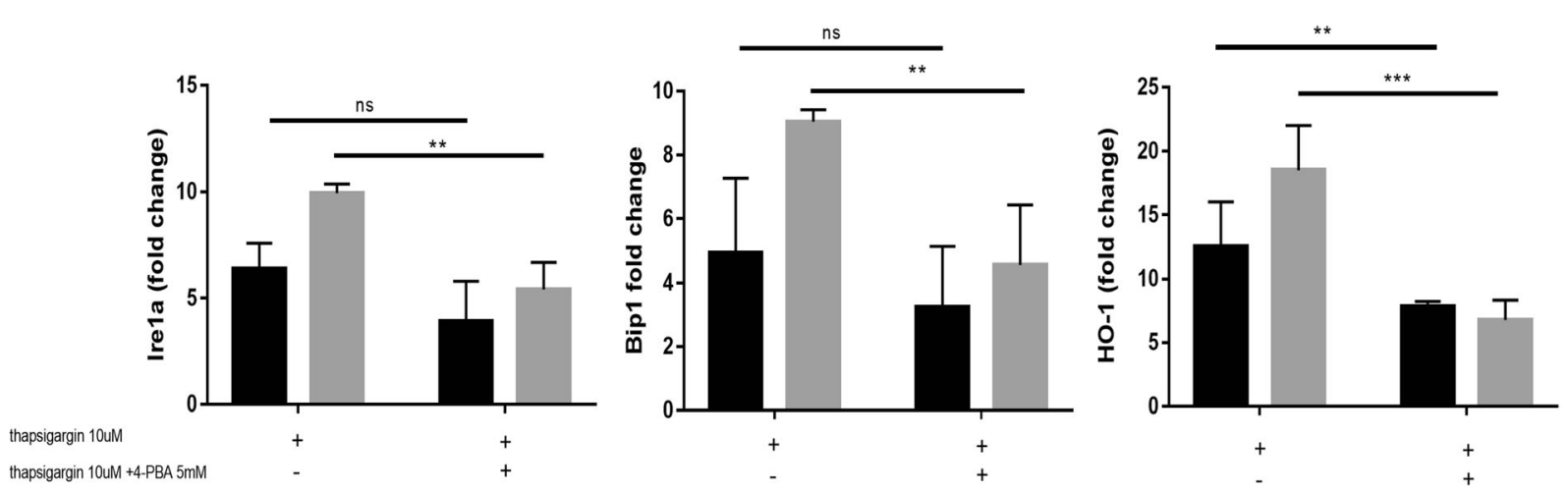

Fig. 4 Gene expression chaperon levels after thapsigargin treatment. Comparison of gene expression chaperon levels of BIP1, Ire1a, and HO-1 in neuoblastoma cell lines treated with thapsigargin $(10 \mu \mathrm{M})$ alone, and in combination with a chemical chaperon 4-PBA $(5 \mathrm{mM})$. The data

\section{Discussion}

In the present study, we showed the role of ALA on the development of chemoresistance in NB cells treated with BTZ. Our data indicate that ALA, in combination with BTZ, acts as chemical chaperone reducing the stress response induced by proteasome inhibition. Firstly, we showed that BTZ significantly reduces cell viability. These data are consistent with previous observations showing that BTZ, a boronic acid dipeptide inhibitor of the $26 \mathrm{~S}$ proteasome [12], downregulates the expression of several antiapoptotic factors as well as it induces caspase- are expressed as mean \pm SEM. The significance of differences between means was analyzed by analysis of variance. $\left({ }^{*} p<0.05\right)\left({ }^{* *} p<0.01\right)$ $(* * * p<0.001)$

dependent apoptosis $[29,30]$. It is well known that proteasome inhibitors induce ER-stress, resulting in a UPR and escalation of ROS that leads to cell death. In cancer cells, HO-1 plays a primary role as essential survival factor, protecting against chemotherapy-induced increase in ROS $[9,11,14]$. These observations were confirmed in the present study. Under our experimental conditions, HO-1 upregulation was observed following BTZ treatment on all tested NB cell lines, suggesting a protective role against BTZ-induced ROS. Concomitantly to HO-1 upregulation, we showed a significant induction of ERstress. Other papers demonstrated that BTZ enhances
Fig. 5 Protein expression chaperon levels of BIP1, Ire1, Ero1, and PDI in neuoblastoma cell lines. Comparison of protein expression of BIP1, Ire1, Ero1, and PDI in neuoblastoma cell lines treated with BTZ $(50 \mathrm{nM})$ alone, with ALA $(100 \mu \mathrm{M})$ alone, and in combination with ALA: BTZ + ALA $(100 \mu \mathrm{M})$. The data are expressed as mean $\pm \mathrm{SEM}$. The significance of differences between means was analyzed by analysis of variance. $(* p<0.05)$ $(* * p<0.01)(* * * p<0.001)$
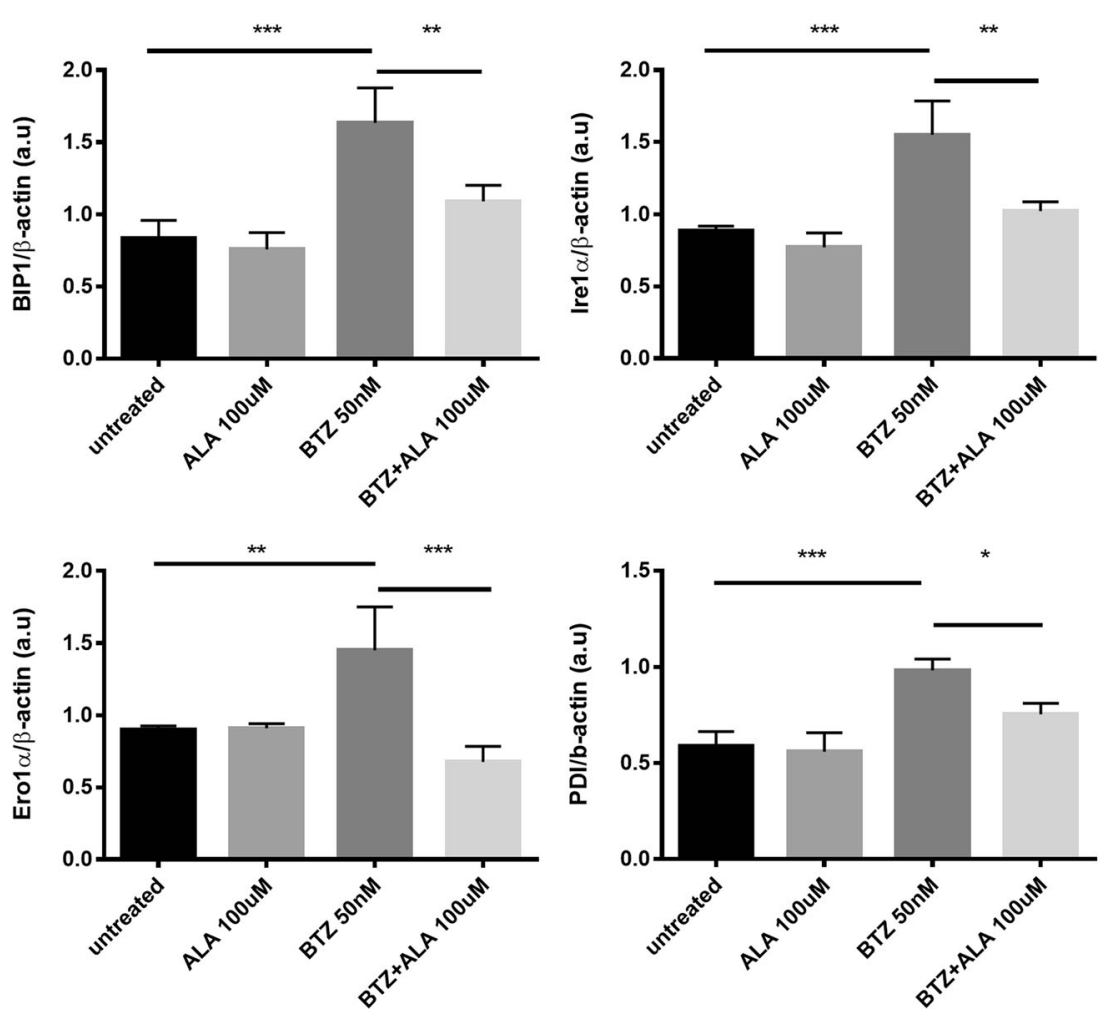
ER-stress [31, 32] and that HO-1 upregulation is dependent on ER-stress [7]. In order to demonstrate the implications of ALA in the mechanism of cell resistance to the cytotoxic effect induced by BTZ, we co-treated NB cells with ALA $100 \mu \mathrm{M}$. Our data shown an increase of cell viability in cells treated with both BTZ and ALA. The cotreatment with $\mathrm{BTZ}+\mathrm{ALA}$ induced a downregulation of HO-1 and ER-stress respect to NB cells treated with BTZ alone. Previous researchers demonstrated that HO-1 is overexpressed in NB cells BTZ-resistant [33], although we shown both a significantly downregulation of HO-1 and cell survival induced by ALA. At the same time and in the same condition, the immunofluorescence assay showed a nuclear localization of HO-1 compared to NB cells treated with BTZ alone. The nuclear localization of HO-1 has been demonstrated in different situations [34, 35] and may serve to upregulate cytoprotective genes against oxidative stress [36]. In this regard, it has been previously demonstrated that nuclear HO-1 is capable to protect leukemic cells from drug-induced toxicity and it could be also implicated as a regulator of DNA repair activities [37, 38]. Thus we supposed that ALA induces cytoprotection in NB cell lines promoting intracellular HO-1 compartmentalization rather than its enzymatic activity. Some researchers [39] showed that nuclear HO-1 modulates the activation of $\mathrm{Nrf} 2$ and induces an adaptive reprogramming that stimulates antioxidant defenses. In order to confirm the pivotal role of HO-1 as chemical chaperone and its antioxidant properties, we treated NB cells with thapsigargin that was able to induce all ERstress proteins and HO-1 and this effect was reversed by addition of 4-PBA. In conclusion, the mechanisms of cytoprotection of ALA against NB cells treated with BTZ seem to be complex. Our hypothesis is that antioxidant properties of ALA under our experimental conditions are not due to upregulation of HO-1 in response to stress induction by BTZ rather its nuclear localization. Recently, some interesting researches [7] demonstrated that protective effect of HO-1 on drug-induced cytotoxicity in leukemic and myeloma cells does not involve its enzymatic by-products, but rather its nuclear translocation following proteolytic cleavage. Furthermore, they showed that HO-1 upregulation is dependent on ER-stress suggesting a link between oxidative stress and UPR. Whether nuclear HO-1 can regulate the transcription of genes implicated in drug resistance wait for further investigations. All these data demonstrated that ALA protects NB cells by stress and damage induced by BTZ since it reduces ER-stress and activates autophagy as mechanism of cell survival. This work confirms the neuroprotective effects of ALA in neurological field and suggests that it should not use in treatment of neuroblastoma disease, since reduces both redox escalation and cellular damage induced by BTZ.

\section{References}

1. Park JR, Eggert A, Caron H (2010) Neuroblastoma: biology, prognosis, and treatment. Hematol Oncol Clin North Am 24(1):65-86. doi:10.1016/j.hoc.2009.11.011

2. Ohira M, Nakagawara A (2010) Global genomic and RNA profiles for novel risk stratification of neuroblastoma. Cancer Sci 101(11): 2295-2301. doi:10.1111/j.1349-7006.2010.01681.x

3. Romano A, Conticello C, Di Raimondo F (2013) Bortezomib for the treatment of previously untreated multiple myeloma. Immunotherapy 5(4):327-352. doi:10.2217/imt.13.14

4. Mujtaba T, Dou QP (2011) Advances in the understanding of mechanisms and therapeutic use of bortezomib. Discov Med 12(67): $471-480$

5. Du BY, Song W, Bai L, Shen Y, Miao SY, Wang LF (2012) Synergistic effects of combination treatment with bortezomib and doxorubicin in human neuroblastoma cell lines. Chemotherapy 58(1):44-51. doi:10.1159/000335603

6. Furfaro AL, Piras S, Passalacqua M, Domenicotti C, Parodi A, Fenoglio D, Pronzato MA, Marinari UM et al (2014) HO-1 upregulation: a key point in high-risk neuroblastoma resistance to bortezomib. Biochim Biophys Acta 1842(4):613-622. doi:10. 1016/j.bbadis.2013.12.008

7. Tibullo D, Barbagallo I, Giallongo C, Vanella L, Conticello C, Romano A, Saccone S, Godos J et al (2016) Heme oxygenase-1 nuclear translocation regulates bortezomibinduced cytotoxicity and mediates genomic instability in myeloma cells. Oncotarget. doi:10. 18632/oncotarget.7563

8. Ryter SW, Choi AM (2009) Heme oxygenase-1/carbon monoxide: from metabolism to molecular therapy. Am J Respir Cell Mol Biol 41(3):251-260. doi:10.1165/rcmb.2009-0170TR

9. Goswami B, Rajappa M, Sharma M, Sharma A (2008) Inflammation: its role and interplay in the development of cancer, with special focus on gynecological malignancies. Int J Gynecol Cancer 18(4):591-599. doi:10.1111/j.1525-1438.2007.01089.x

10. Abe M (2011) Guest editorial: understanding the pathogenesis and the evolving treatment paradigm for multiple myeloma in the era of novel agents. Int J Hematol 94(4):307-309. doi:10.1007/s12185011-0950-4

11. Meister S, Schubert U, Neubert K, Herrmann K, Burger R, Gramatzki M, Hahn S, Schreiber S et al (2007) Extensive immunoglobulin production sensitizes myeloma cells for proteasome inhibition. Cancer Res 67(4):1783-1792. doi:10.1158/0008-5472. CAN-06-2258

12. Teicher BA, Ara G, Herbst R, Palombella VJ, Adams J (1999) The proteasome inhibitor PS-341 in cancer therapy. Clin Cancer Res 5(9):2638-2645

13. Richardson PG, Baz R, Wang M, Jakubowiak AJ, Laubach JP, Harvey RD, Talpaz M, Berg D et al (2014) Phase 1 study of twice-weekly ixazomib, an oral proteasome inhibitor, in relapsed/ refractory multiple myeloma patients. Blood 124(7):1038-1046. doi:10.1182/blood-2014-01-548826

14. Salerno L, Pittala V, Romeo G, Modica MN, Siracusa MA, Di Giacomo C, Acquaviva R, Barbagallo I et al (2013) Evaluation of novel aryloxyalkyl derivatives of imidazole and 1,2,4-triazole as heme oxygenase-1 (HO-1) inhibitors and their antitumor properties. Bioorg Med Chem 21(17):5145-5153. doi:10.1016/j.bmc.2013.06.040

15. Goodman AI, Choudhury M, da Silva JL, Schwartzman ML, Abraham NG (1997) Overexpression of the heme oxygenase gene in renal cell carcinoma. Proc Soc Exp Biol Med 214(1):54-61

16. Becker JC, Fukui H, Imai Y, Sekikawa A, Kimura T, Yamagishi H, Yoshitake N, Pohle T et al (2007) Colonic expression of heme oxygenase- 1 is associated with a better long-term survival in patients with colorectal cancer. Scand J Gastroenterol 42(7):852-858. doi:10.1080/00365520701192383 
17. Yamada T, Hashida K, Takarada-Iemata M, Matsugo S, Hori O (2011) Alpha-lipoic acid (LA) enantiomers protect SH-SY5Y cells against glutathione depletion. Neurochem Int 59(7):1003-1009. doi:10.1016/j.neuint.2011.09.005

18. Bramanti V, Tomassoni D, Bronzi D, Grasso S, Curro M, Avitabile M, Li Volsi G, Renis M et al (2010) Alpha-lipoic acid modulates GFAP, vimentin, nestin, cyclin D1 and MAP-kinase expression in astroglial cell cultures. Neurochem Res 35(12):2070-2077. doi:10. 1007/s11064-010-0256-6

19. Giallongo C, Parrinello N, Tibullo D, La Cava P, Romano A, Chiarenza A, Barbagallo I, Palumbo GA et al (2014) Myeloid derived suppressor cells (MDSCs) are increased and exert immunosuppressive activity together with polymorphonuclear leukocytes (PMNs) in chronic myeloid leukemia patients. PLoS One 9(7): e101848. doi:10.1371/journal.pone.0101848

20. Giallongo C, Tibullo D, La Cava P, Branca A, Parrinello N, Spina P, Stagno F, Conticello C et al (2011) BRIT1/MCPH1 expression in chronic myeloid leukemia and its regulation of the G2/M checkpoint. Acta Haematol 126(4):205-210. doi:10.1159/000329911

21. Anfuso CD, Motta C, Giurdanella G, Arena V, Alberghina M, Lupo G (2014) Endothelial PKCalpha-MAPK/ERK-phospholipase A2 pathway activation as a response of glioma in a triple culture model. A new role for pericytes? Biochimie 99:77-87. doi:10.1016/j. biochi.2013.11.013

22. Avola R, Di Tullio MA, Fisichella A, Tayebati SK, Tomassoni D (2004) Glial fibrillary acidic protein and vimentin expression is regulated by glucocorticoids and neurotrophic factors in primary rat astroglial cultures. Clin Exp Hypertens 26(4):323-333

23. Bramanti V, Bronzi D, Tomassoni D, Li Volti G, Cannavo G, Raciti G, Napoli M, Vanella A et al (2008) Effect of choline-containing phospholipids on transglutaminase activity in primary astroglial cell cultures. Clin Exp Hypertens 30(8):798-807. doi:10.1080/ 10641960802563576

24. Bramanti V, Grasso S, Tomassoni D, Traini E, Raciti G, Viola M, Li Volti G, Campisi A et al (2015) Effect of growth factors and steroid hormones on heme oxygenase and cyclin D1 expression in primary astroglial cell cultures. J Neurosci Res 93(3):521-529. doi:10.1002/ jnr.23506

25. Scuderi MR, Anfuso CD, Lupo G, Motta C, Romeo L, Guerra L, Cappellani A, Ragusa N et al (2008) Expression of $\mathrm{Ca}(2+)$-independent and $\mathrm{Ca}(2+)$-dependent phospholipases $\mathrm{A}(2)$ and cyclooxygenases in human melanocytes and malignant melanoma cell lines. Biochim Biophys Acta 1781(10):635-642. doi:10.1016/j. bbalip.2008.07.007

26. Lupo G, Anfuso CD, Ragusa N, Tirolo C, Marchetti B, Gili E, La Rosa C, Vancheri C (2007) Activation of cytosolic phospholipase $\mathrm{A} 2$ and 15-lipoxygenase by oxidized low-density lipoproteins in cultured human lung fibroblasts. Biochim Biophys Acta 1771(4): 522-532. doi:10.1016/j.bbalip.2007.01.014

27. Bramanti V, Tomassoni D, Grasso S, Bronzi D, Napoli M, Campisi A, Li Volti G, Ientile R et al (2012) Cholinergic precursors modulate the expression of heme oxigenase-1, p21 during astroglial cell proliferation and differentiation in culture. Neurochem Res 37(12): 2795-2804. doi:10.1007/s11064-012-0873-3
28. Giurdanella G, Anfuso CD, Olivieri M, Lupo G, Caporarello N, Eandi CM, Drago F, Bucolo C et al (2015) Aflibercept, bevacizumab and ranibizumab prevent glucose-induced damage in human retinal pericytes in vitro, through a PLA2/COX-2/ VEGF-A pathway. Biochem Pharmacol 96(3):278-287. doi:10. 1016/j.bcp.2015.05.017

29. Hideshima T, Chauhan D, Richardson P, Mitsiades C, Mitsiades N, Hayashi T, Munshi N, Dang L et al (2002) NF-kappa B as a therapeutic target in multiple myeloma. J Biol Chem 277(19):1663916647. doi:10.1074/jbc.M200360200

30. Almond JB, Cohen GM (2002) The proteasome: a novel target for cancer chemotherapy. Leukemia 16(4):433-443. doi:10.1038/sj. leu. 2402417

31. Obeng EA, Carlson LM, Gutman DM, Harrington WJ Jr, Lee KP, Boise LH (2006) Proteasome inhibitors induce a terminal unfolded protein response in multiple myeloma cells. Blood 107(12):49074916. doi:10.1182/blood-2005-08-3531

32. Chien W, Ding LW, Sun QY, Torres-Fernandez LA, Tan SZ, Xiao J, Lim SL, Garg M et al (2014) Selective inhibition of unfolded protein response induces apoptosis in pancreatic cancer cells. Oncotarget 5(13):4881-4894. doi:10.18632/oncotarget.2051

33. Furfaro AL, Piras S, Domenicotti C, Fenoglio D, De Luigi A, Salmona M, Moretta L, Marinari UM et al (2016) Role of Nrf2, HO-1 and GSH in neuroblastoma cell resistance to bortezomib. PLoS One 11(3):e0152465. doi:10.1371/journal.pone.0152465

34. Ghobrial IM, Munshi NC, Harris BN, Shi P, Porter NM, Schlossman RL, Laubach JP, Anderson KC et al (2011) A phase I safety study of enzastaurin plus bortezomib in the treatment of relapsed or refractory multiple myeloma. Am J Hematol 86(7): 573-578. doi:10.1002/ajh.22048

35. Li Volti G, Ientile R, Abraham NG, Vanella A, Cannavo G, Mazza F, Curro M, Raciti G et al (2004) Immunocytochemical localization and expression of heme oxygenase-1 in primary astroglial cell cultures during differentiation: effect of glutamate. Biochem Biophys Res Commun 315(2):517-524. doi:10.1016/j.bbrc.2004.01.090

36. Lin Q, Weis S, Yang G, Weng YH, Helston R, Rish K, Smith A, Bordner J et al (2007) Heme oxygenase-1 protein localizes to the nucleus and activates transcription factors important in oxidative stress. J Biol Chem 282(28):20621-20633. doi:10.1074/jbc. M607954200

37. Tibullo D, Barbagallo I, Giallongo C, La Cava P, Parrinello N, Vanella L, Stagno F, Palumbo GA et al (2013) Nuclear translocation of heme oxygenase-1 confers resistance to imatinib in chronic myeloid leukemia cells. Curr Pharm Des 19(15):2765-2770

38. Gandini NA, Fermento ME, Salomon DG, Blasco J, Patel V, Gutkind JS, Molinolo AA, Facchinetti MM et al (2012) Nuclear localization of heme oxygenase- 1 is associated with tumor progression of head and neck squamous cell carcinomas. Exp Mol Pathol 93(2):237-245. doi:10.1016/j.yexmp.2012.05.001

39. Biswas C, Shah N, Muthu M, La P, Fernando AP, Sengupta S, Yang G, Dennery PA (2014) Nuclear heme oxygenase-1 (HO-1) modulates subcellular distribution and activation of $\mathrm{Nrf} 2$, impacting metabolic and anti-oxidant defenses. J Biol Chem 289(39):2688226894. doi:10.1074/jbc.M114.567685 